\title{
Efecto del vaporizado inicial en el secado artificial de madera de coiguie de Magallanes (Nothofagus betuloides)*
}

Effect of steaming in the artificial drying of coigüe of Magallanes wood (Nothofagus betuloides)

ROBERTO JUACIDA P ${ }^{1}$., LUIS INZUNZA D ${ }^{1}$., CRISTIAN BARRIA C ${ }^{2}$.

${ }^{1}$ Universidad Austral de Chile, Instituto de Tecnología de Productos Forestales, Casilla 567, Valdivia, Chile. ${ }^{2}$ Ingeniero Forestal.

\begin{abstract}
SUMMARY
The work consisted on subjecting Nothofagus betuloides (Mirb.) Oerst., sawed wood at three different levels of steaming on the beginning of drying process $(4,8$ and 10 hours) and then to a standard conventional drying. The effect of steaming on wood quality and the time required to obtain the desired moisture content on wood (8\%) were evaluated. The raw material was provided by daily production of an industrial plant, located in the Punta Arenas city, southern Chile. The selection of sawed wood was randomized to obtain a total of 150 fresh-sawedstate samples of 1 " $\times 6$ " $\times 2.5 \mathrm{~m}$, corresponding to $1.5 \mathrm{~m}^{3}$. The experiments was taken out in the wood drier equipment of Forestry Faculty of Universidad Austral de Chile. Results show that increasing the time of presteaming treatment improves wood quality and reduces final time of drying, specially 10 hours that presented better results on quality. The time of drying that provides a total time of 302 hours or $8 \%$ less than control.
\end{abstract}

Key words: coigüe de Magallanes, drying, steaming.

\section{RESUMEN}

El trabajo consistió básicamente en someter madera aserrada de Nothofagus betuloides (Mirb.) Oerst. a tres distintos niveles de vaporizado inicial $(4,8$ y 10 horas, respectivamente) y a un posterior proceso de secado artificial convencional único. Se evaluó el efecto del vaporizado inicial sobre la calidad de la madera obtenida y el tiempo de secado requerido para alcanzar el contenido de humedad deseado de un $8 \%$. La madera utilizada para los ensayos se obtuvo de la producción diaria de una planta industrial, ubicada en la ciudad de Punta Arenas, XII Región, Chile. Se seleccionó en forma aleatoria un total de 150 tablas, recién aserradas y en estado verde de 1" x 6" x $2,5 \mathrm{~m}$, equivalente a $1,5 \mathrm{~m}^{3}$. Los ensayos se efectuaron en el secador experimental de la Facultad de Ciencias Forestales de la Universidad Austral de Chile. Los resultados obtenidos indican que a medida que se aumentan las horas de pretratamiento, en el rango seleccionado, se mejora en cierta medida la calidad y tiempo final del secado, siendo el vaporizado de 10 horas el que otorgó mejores resultados. El índice que más incidió en desclasificar la madera fue el alabeo, siendo la encorvadura el defecto más importante. Con respecto a grietas, fueron principalmente superficiales, no evidenciándose internas. El tiempo total de secado fue de 302 horas, reduciendo según el testigo en un $8 \%$ el tiempo total.

Palabras clave: coigüe de Magallanes, secado, vaporizado inicial.

\footnotetext{
* Proyecto financiado por Forestal Trillium de Chile Ltda.
} 


\section{INTRODUCCION}

La superficie boscosa de la XII Región está representada principalmente por dos especies como son (lenga) Nothofagus pumilio (Poepp. et Endl.) Krasser y (coigüe de Magallanes) Nothofagus betuloides (Mirb.) Oerst., las que han sido la base de la producción forestal y por las que en la década de los noventa han surgido fuertes inversiones. Esta última especie, además de ser un árbol endémico de los bosques subantárticos, a nivel nacional ocupa como tipo forestal la tercera posición en superficie de bosque nativo distribuida entre la Décima y Duodécima Regiones. Dentro de sus características propias presenta una madera homogénea, bella, de buenas propiedades mecánicas, durable y sobre todo de buena trabajabilidad, lo que posibilita una amplia gama de usos, destacándose principalmente la mueblería, producto final con un alto valor agregado.

Sin embargo, es una especie que presenta dificultades para ser secada artificialmente, lo que ha llevado a estudiar alternativas complementarias que permitan reducir los tiempos y mejorar la calidad de la madera al término del proceso. Dentro de estas alternativas se encuentra la aplicación de un vaporizado inicial a la madera, pretratamiento recomendado como técnicamente ventajoso $(1,2)$.

Como objetivo general se pretende evaluar el efecto del tiempo de aplicación de vaporizado inicial sobre la calidad de la madera obtenida y el tiempo de secado requerido para alcanzar el contenido de humedad deseado. Como objetivo específico se plantea probar tres distintos niveles de tiempo de vaporizado inicial como pretratamiento asociados a un programa de secado base y medir, por un lado, en cuál de ellos la calidad de madera es mejor y, por otro, el tiempo requerido para alcanzar el contenido de humedad final necesario.

\section{MATERIAL Y METODOS}

La madera aserrada necesaria para llevar a efecto el presente estudio corresponde a coigüe de Magallanes proveniente de la XII Región (Chile). Corresponde a madera escogida al azar, recién aserrada, verde y de dimensiones definidas, proveniente de la producción diaria de un aserradero ubicado en la ciudad de Punta Arenas.
La muestra fue extraída de un total de 80 trozos, con rangos diamétricos entre 30 a $45 \mathrm{~cm}$ y 3,6 m de largo, los que eran sanos, rectos y con una clara diferenciación entre albura y duramen en la sección transversal del trozo. Se utilizó un secador piloto marca Hildebrand HB 75/sp de aproximadamente $0,3 \mathrm{~m}^{3}$ de capacidad, el que posee un rango de temperatura entre 0 y $130{ }^{\circ} \mathrm{C}$, un dispositivo de vaporizado eléctrico, una velocidad del viento unidireccional de $3 \mathrm{~m} / \mathrm{s}$ y un moderno sistema computacional de control del proceso del secado planteado por Mühlbock. Además, se utilizaron balanzas analíticas, diales de comparación, pie de metro y estufas. La madera de coigüe de Magallanes se somete a tres distintos procesos de vaporizado inicial $(4,8$ y 10 horas) e inmediatamente es secada con un programa convencional único. La calidad del vapor fue saturado a $40{ }^{\circ} \mathrm{C}$. Como referencia se realizó un secado de la madera sin considerar el tratamiento de vaporizado.

Una vez preparadas las piezas de secado se confeccionó el castillo con un total de 40 piezas de dimensiones de 1" x 6" x $1,1 \mathrm{~m}$ distribuidas en cuatro columnas y 10 filas; entre cada fila se ubicaron cuatro separadores equidistantes entre sí. En tanto, sobre la carga se colocó un sobrepeso aproximado de $350 \mathrm{~kg} / \mathrm{m}^{2}$. En la parte superior, intermedia y inferior del castillo se colocaron cinco piezas testigos de humedad, a fin de obtener una representatividad de éste $(3,4)$.

Se utilizó un programa de secado basado en la pérdida de humedad de la madera, elaborado y probado a escala industrial por Inzunza (5), tomando en consideración recomendaciones realizadas en otros estudios $(6,7,8)$. El programa contó con cuatro etapas: la de pretratamiento con sus respectivos tiempos; la de secado sobre el PSF; la de secado bajo PSF y, por último, la etapa de acondicionamiento final. En relación con la temperatura, ésta fue relativamente baja con un mínimo de $40{ }^{\circ} \mathrm{C}$ y un máximo de $55^{\circ} \mathrm{C}$, con el propósito de alcanzar una humedad de equilibrio final de $8 \%$.

El control del proceso de secado se realizó a partir de esquemas descritos y aplicados por otros autores $(2,9,10)$. Se hicieron controles antes, durante y posteriores al secado, utilizando las normas chilenas específicas para cada determinación y que se mencionan en la bibliografía (11, $12,13,14)$. 
Tanto para evaluar las tensiones de secado como la intensidad del colapso se utilizó la metodología descrita por Welling y Alvarez (15).

La determinación cuantitativa de las tensiones de secado se realizó utilizando el método Trada que mide el grado de flexión de los dientes en probetas de tenedor y según ello califica la tensión en tres grados de deformación: ligero, moderado y fuerte.

La intensidad del colapso se midió utilizando el método de comparar las diferencias de espesores mayor y menor de la sección transversal afectada; las calidades resultantes se especifican en "leve", pérdida de volumen menor o igual a 2 $\mathrm{mm}$ y que desaparece con cepillado superficial; "moderado", pérdida de volumen menor o igual a $3 \mathrm{~mm}$ y que desaparece con cepillado intenso, y "fuerte", pérdida considerable de volumen, mayor a $5 \mathrm{~mm}$.

Para evaluar el deterioro causado por las grietas y alabeos, se utilizó la clave que considera cuatro grados de intensidad (cuadro 1), aplicada en trabajos anteriores $(2,3,6)$.

\section{CUADRO 1}

Clasificación de grietas y alabeos.

Classification of checking and warp.

\begin{tabular}{|lc|}
\hline Niveles & Intensidad \\
\hline SD (Sin defecto) & 0,0 \\
L (Leve) & 0,5 \\
LI (Leve intenso) & 1,0 \\
I (Intenso) & 1,5 \\
\hline
\end{tabular}

La calidad del secado se estimó como la suma ponderada de las medias estadísticas de los defectos evaluados. Se obtuvo un subíndice de grieta y uno de alabeo, los que sumados dieron el índice final «I» (cuadro 2).

\section{RESULTADOS Y DISCUSION}

Un análisis comparativo entre los tres distintos pretratamientos incluyendo además el secado testigo se presenta en el cuadro 3, con el propósito de evaluar el comportamiento experimentado tanto por la calidad final de la madera como el tiempo total de secado. Se puede observar que la

\section{CUADRO 2}

Clasificación de calidad y condiciones de secado. Classification of quality and drying conditions.

\begin{tabular}{|lcl|}
\hline Calidad del secado & Indice & $\begin{array}{l}\text { Condiciones } \\
\text { de secado }\end{array}$ \\
\hline $\begin{array}{l}\text { Excelente } \\
\text { Muy buena }\end{array}$ & $\begin{array}{c}\text { hasta } 0,5 \\
\text { hasta } 1,0\end{array}$ & Adecuadas \\
Regular & hasta 1,5 & \\
Menos que regular & hasta 2,0 & Poco adecuadas \\
Defectuosa & hasta 3,0 & \\
Mala & hasta 5,0 & Inadecuadas \\
Muy mala & más 5,0 & \\
\hline
\end{tabular}

Fuente: Figueroa (2).

calidad de la madera mejoró a medida que aumentan las horas de pretratamiento. Mejoró gradualmente desde un índice 3,4 en el caso del testigo que corresponde a un secado de mala calidad y una condición inadecuada, pasando por índices 2,7 y 2,2 para el caso de 4 y 8 horas de pretratamiento que corresponden a secados de calidad defectuosa y condición inadecuada. El menor índice de 1,9 se obtiene en el pretratamiento de 10 horas que indica un secado de calidad menos que regular y una condición poco adecuada. Además, se observa que el subíndice que más incidió en la calidad final de madera fue el de alabeo, principalmente por efecto de la encorvadura, teniendo un comportamiento similar en tendencia y magnitud. Esto no ocurre para el subíndice grieta. En relación con el tiempo total de secado, se observa que éste disminuyó a medida que aumentaron las horas de pretratamiento, mejorando aproximadamente en un $8 \%$ si lo comparamos con el testigo.

En las figuras 1, 2 y 3 se presentan las curvas de secado para todos los tratamientos aplicados, detallando sólo aquel que arrojó los mejores resultados

En consideración a que el pretratamiento de 10 horas presentó el mejor comportamiento tanto en la calidad final de la madera como en el tiempo total de secado, a continuación se realiza un resumen detallado de sus resultados. 


\section{CUADRO 3}

Efecto del pretratamiento en la calidad y tiempo total de secado.

Effect pre-treatment on the full time and quality of drying.

\begin{tabular}{|lcccc|}
\hline & \multicolumn{5}{c|}{ Pretratamientos (h) } \\
\cline { 2 - 5 } Item evaluación & 4 & 8 & 10 & Testigo \\
\hline Subíndice de grieta & 0,05 & 0,11 & 0,05 & 0,12 \\
Subíndice de alabeo & 2,6 & 2,1 & 1,9 & 3,3 \\
Calidad final & 2,65 & 2,21 & 1,95 & 3,4 \\
Tiempo total (h) & 315 & 311 & 302 & 320 \\
CH inicial (\%) & 52,8 & 70 & 63,3 & 64,9 \\
CH final (\%) & 6,9 & 9,1 & 6,8 & 7,9 \\
\hline
\end{tabular}



Figura 1. Diagrama de secado sin vapor inicial. Diagram of drying without initial steam.

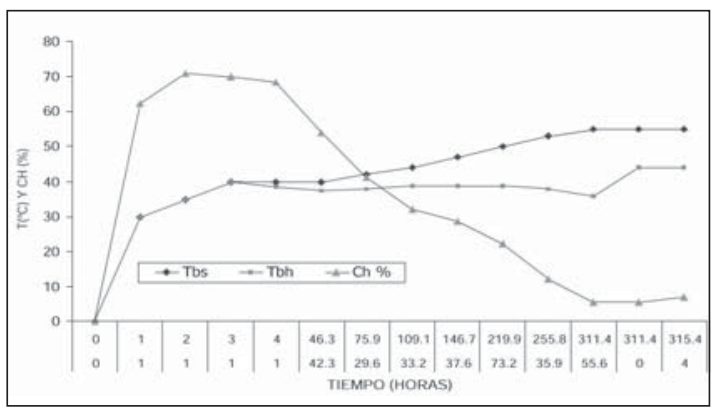

Figura 2. Diagrama de secado con 4 horas de vapor. Diagram of drying for 4 hours of steam.

\section{CONTROL DEL PROCESO DE SECADO}

Programa aplicado: En la figura 4 se presenta el diagrama de secado realmente aplicado y contro-

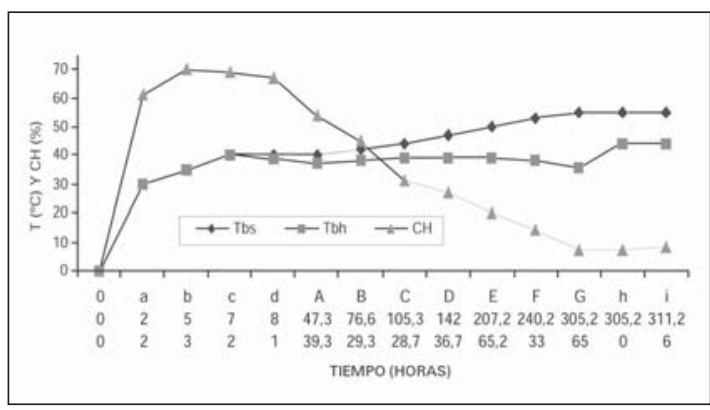

Figura 3. Diagrama de secado con 8 horas de vapor. Diagram of drying with 8 hours of steam.

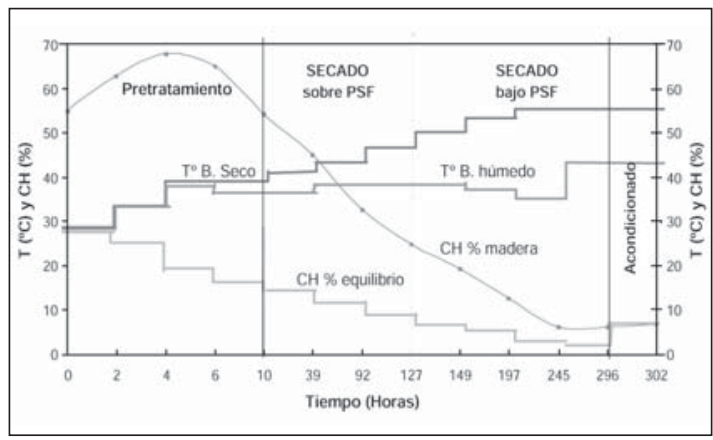

Figura 4. Diagrama de secado con 10 horas de vapor. Diagram of drying with 10 hours of steam.

lado por medio de las piezas testigos y los respectivos bulbos de medición de temperatura en las distintas etapas.

La etapa de pretratamiento tuvo una duración de 10 horas, subiendo gradualmente las temperaturas hasta llegar a los $40{ }^{\circ} \mathrm{C}$. El contenido de humedad inicial de las piezas testigo fue de $55 \%$, la que aumentó hasta un $68 \%$ por efecto del vaporizado. Durante la etapa de secado, sobre el punto de saturación de fibras, se elevó lentamente la temperatura llegando a $44{ }^{\circ} \mathrm{C}$, con una diferencia psicrométrica inicial de $2,5^{\circ} \mathrm{C}$, la que aumentó hasta llegar a $5{ }^{\circ} \mathrm{C}$, momento en que las piezas testigos alcanzaron un contenido de humedad alrededor del 30\%. El contenido de humedad de la madera se redujo hasta un $32,7 \%$. En la etapa de secado, bajo el punto de saturación de las fibras, se llegó a una temperatura máxima del bulbo seco de $55^{\circ} \mathrm{C}$. La diferencia psicrométrica se aumentó hasta alcanzar $19^{\circ} \mathrm{C}$. El contenido de humedad de la madera se redujo a $6,0 \%$. El acondicionamiento duró 6 horas y se alcanzó un 6,4\% de humedad de la madera. 
CONTROL DE CALIDAD DEL SECADO

Contenido de humedad de la carga: Se propuso un contenido de humedad final de $8 \%$ y se alcanzó un promedio de $6,8 \%$ con una desviación estándar de 0,5. El contenido de humedad inicial de la madera fue de $63,3 \%$ con un mínimo de $46 \%$ y un máximo de $100 \%$. El contenido de humedad en el interior de las tablas fue un 1,2\% superior al de la superficie.

Contracción: Se observa que la contracción en el ancho de una madera de corte tangencial es un $1,9 \%$ mayor que la correspondiente al ancho de un corte radial. Por otra parte, la contracción en el espesor de una madera de corte radial es un $2,7 \%$ mayor que la correspondiente al espesor de un corte tangencial (cuadro 4 ).

Tensiones de secado: En los controles realizados una vez finalizado el secado, se detectó cierto grado de tensión. De acuerdo al método Trada, un $87 \%$ de las piezas sufrió tensiones moderadasintensas (80\% "moderadas" y un $7 \%$ "intensas") y un $13 \%$ "leves".

Colapso: El número de piezas afectadas por colapso fue de un $25 \%$. De acuerdo a la clasificación de calidad descrita en la metodología, un $80 \%$ de las piezas fueron afectadas por un colapso "leve", un $10 \%$ "moderado" y el $10 \%$ restante, "fuerte".

Agrietamiento: El 10\% de la carga total presentó grietas en diversos grados. El agrietamiento superficial resultó ser el único defecto de mayor

\section{CUADRO 4}

Contracción (\%) según tipo de corte en madera. Contraction (\%) acording type of cut in wood.

\begin{tabular}{|lccccc|}
\hline & \multicolumn{2}{c}{ Tangencial } & & \multicolumn{2}{c|}{ Radial } \\
\cline { 2 - 3 } \cline { 6 - 7 } & Ancho & Espesor & & Ancho & Espesor \\
\hline Promedio & 6,0 & 3,4 & & 4,1 & 6,1 \\
Máximo & 7,8 & 6,7 & & 8,6 & 6,4 \\
Mínimo & 3,6 & 0,3 & & 5,5 & 3,0 \\
D. estándar & 1,1 & 3,2 & & 1,1 & 2,0 \\
Volumétrica & \multicolumn{2}{c}{9,4} & & \multicolumn{2}{c}{10,2} \\
\hline
\end{tabular}

frecuencia, destacándose además la no existencia de grietas internas ni en los extremos. En el caso de las grietas superficiales éstas aparecieron mayoritariamente alrededor de los nudos. El índice de agrietamiento fue de 0,05 (cuadro 5).

\section{CUADRO 5}

Cuantificación de grietas y su ubicación en la pieza. Quantification of crack and this ubication in the piece.

\begin{tabular}{|lcccc|}
\hline Nivel de & \multicolumn{4}{c|}{ Número de Grietas } \\
\cline { 2 - 5 } clasificación & Superficial & Internas & Extremos & Factor \\
\hline Sin defecto & 36 & 40 & 40 & 0 \\
Leve & 4 & 0 & 0 & 2 \\
Leve-intenso & 0 & 0 & 0 & 0 \\
Intenso & 0 & 0 & 0 & 0 \\
\hline
\end{tabular}

Alabeos: Dentro de los distintos tipos de alabeos, la encorvadura y la acanaladura fueron los de mayor frecuencia con un 70 y $55 \%$ respectivamente. La mayor intensidad de defecto se registró también en la encorvadura. El índice de alabeo fue de 1,9 (cuadro 6).

Se observaron varias piezas con dos o más defectos a la vez, siendo la combinación de mayor frecuencia la encorvadura con la torcedura y que se registraron principalmente en piezas de corte radial. La calidad final del secado, aplicando un pretratamiento de 10 horas, considerando los índices (grietas + alabeos) de 1,9, resultó ser menos que regular, enmarcándolo dentro de un secado poco adecuado. Se puede apreciar que los alabeos siguen siendo el elemento de desclasificación más incidente en la calidad final del secado.

\section{DURACION DEL PROCESO DE SECADO}

En la figura 4 se observa que el tiempo total de duración del programa de secado fue de 302 horas, que equivalen a 12,6 días, considerando en ello el proceso de calentamiento y acondicionado. Presenta una reducción del $8 \%$ en relación con el tiempo del testigo. 


\section{CUADRO 6}

Frecuencia de alabeos y su incidencia.

Frequency of warp and this incidence.

\begin{tabular}{|lcccc|}
\hline \multirow{2}{*}{$\begin{array}{l}\text { Grado de } \\
\text { deformación }\end{array}$} & \multicolumn{4}{c|}{ Frecuencia de la carga afectada por alabeos (n) } \\
\cline { 2 - 5 } & Arqueadura & Encorvadura & Torcedura & Acanaladura \\
\hline Sin defecto & 24 & 12 & 20 & 18 \\
Alabeo leve & 14 & 6 & 4 & 12 \\
Alabeo leve-intenso & 1 & 6 & 14 & 10 \\
Alabeo intenso & 1 & 16 & 2 & 0 \\
\hline
\end{tabular}

\section{CONCLUSIONES}

Existe una mejoría tanto en la calidad final como el tiempo total de secado, a medida que aumentan las horas de pretratamiento, obteniendo al cabo de las 10 horas los siguientes resultados:

- Un índice final de secado de 1,9. Valor que otorga una madera de calidad de secado menos que regular y una condición poco adecuada.

- El subíndice alabeo fue el que más incidió en la calidad final de la madera, a través de la encorvadura.

- El tiempo total de secado fue de 302 horas (12,6 días), logrando reducir aproximadamente en un $8 \%$ el tiempo, en relación con el testigo.

- El contenido de humedad final fue de $6,8 \%$, valor inferior al establecido como objetivo.

El programa de secado asociado a los tres distintos pretratamientos debe ser considerado como regular, lo que da pie a continuar con las investigaciones y mejoramiento sobre la base del comportamiento experimentado.

\section{BIBLIOGRAFIA}

(1) JUACIDA, R. El vaporizado como pretratamiento para la impregnación de durmientes de coigüe, Nothofagus dombeyi Mirb. Oerst. Tesis Ingeniería Forestal, Universidad Austral de Chile, Valdivia (Chile), 1969, 59 p.

(2) FIGUEROA, R. Secado artificial de madera de coigüe (Nothofagus dombeyi (Mirb.) Oerst), previa aplicación de un pretratamiento de inmersión en agua caliente. Tesis Ingeniería Forestal, Universidad Austral de Chile, Valdivia (Chile), 1991, 68 p.

(3) RAMIREZ, J. Comportamiento del pino oregón (Pseudotsuga menziesii (Mirb) Franco) frente al secado artificial. Tesis Ingeniería Forestal, Universidad Austral de Chile, Valdivia (Chile), 1984, 87 p.
(4) PAN DE LEIVA, E. Secado de renovales de raulí (Nothofagus alpina (Poepp. et Endl.) Oerst). Tesis Magíster en Ciencias, Universidad Austral de Chile, Valdivia (Chile), 1995, 103 p.

(5) INZUNZA, L. Técnicas de secado artificial de lenga y coigüe de Magallanes. Informe Convenio UACh Trillium, 2001, 26 p.

(6) MUÑOZ, R. Ensayo con tres programas de secado artificial en cinco especies nativas latifoliadas. Tesis de Ingeniería Forestal, Universidad Austral de Chile, Valdivia (Chile), 1972, $112 \mathrm{p}$.

(7) SALGADO, S. Aplicación y análisis de tres programas de secado artificial con madera de lenga (Nothofagus pumilio). Tesis Ingeniería Forestal, Universidad Austral de Chile, Valdivia (Chile), 1974, 49 p.

(8) BAHAMONDES, R. Proposición de un programa de secado artificial de madera de coigüe de Magallanes (Nothofagus betuloides Mirb. Oerst) de hasta 1.5" de espesor, previa aplicación y análisis de tres programas tentativos. Tesis Ingeniería Forestal. Universidad Austral de Chile, Valdivia (Chile), 1976, 66 p.

(9) FUENTES, M. Secado de la madera aserrada de Pinus radiata D. Don, impregnada con sales hidrosolubles CCA. Tesis Magíster en Ciencias, Universidad Austral de Chile, Valdivia (Chile), 1994, 162 p.

(10) SALDÍVAR, E. Aserrío y secado de tres cultivares de Populus x euramericana (Dode) Guinier creciendo en la Provincia de Valdivia. Tesis Ingeniería Forestal, Universidad Austral de Chile, Valdivia (Chile), 1999, 98 p.

(11) CHILE. INSTITUTO NACIONAL DE NORMALIZACIÓN. NCh 992 Eof 72. Madera. Defectos a considerar en la clasificación. Terminología y métodos de medición. Santiago (Chile), 1972, 22 p.

(12) —. Maderas. NCh 176-1 OF-83. Determinación de la humedad. Santiago (Chile), 1983, 7 p.

(13) - NCh 176-3 CR-83. Maderas. Determinación de la contracción radial y tangencial. Santiago (Chile), 1983, 9 p.

(14) - NCh 176-2 OF-86. Maderas. Determinación de la densidad. Santiago (Chile), 1986, 11 p.

(15) WELliNG, J., ALVAREZ, H. Definición y evaluación de la calidad de secado de la madera aserrada. Seminario Avances en Tecnología de la Madera, Universidad Austral de Chile, Valdivia (Chile), 1964, 28 p. 